\title{
Differential secretion of the mutated protein is a major component affecting phenotypic severity in CRLF1-associated disorders
}

\author{
Jana Herholz ${ }^{1,10}$, Alessandra Meloni ${ }^{2,10}$, Mara Marongiu ${ }^{2}$, Francesca Chiappe ${ }^{2,3}$, Manila Deiana ${ }^{2}$, \\ Carmen Roche Herrero ${ }^{4}$, Giuseppe Zampino ${ }^{5}$, Hanan Hamamy ${ }^{6}$, Yusra Zalloum ${ }^{7}$, Per Erik Waaler ${ }^{8}$, \\ Giangiorgio Crisponi ${ }^{9}$, Laura Crisponi ${ }^{\star, 2}$ and Frank Rutsch ${ }^{1}$
}

Crisponi syndrome (CS) and cold-induced sweating syndrome type 1 (CISS1) are disorders caused by mutations in CRLF1. The two syndromes share clinical characteristics, such as dysmorphic features, muscle contractions, scoliosis and cold-induced sweating, with CS patients showing a severe clinical course in infancy involving hyperthermia, associated with death in most cases in the first years of life. To evaluate a potential genotype/phenotype correlation and whether CS and CISS1 represent two allelic diseases or manifestations at different ages of the same disorder, we carried out a detailed clinical analysis of 19 patients carrying mutations in CRLF1. We studied the functional significance of the mutations found in CRLF1, providing evidence that phenotypic severity of the two disorders mainly depends on altered kinetics of secretion of the mutated CRLF1 protein. On the basis of these findings, we believe that the two syndromes, CS and CISS1, represent manifestations of the same disorder, with different degrees of severity. We suggest renaming the two genetic entities CS and CISS1 with the broader term of Sohar-Crisponi syndrome.

European Journal of Human Genetics (2011) 19, 525-533; doi:10.1038/ejhg.2010.253; published online 16 February 2011

Keywords: Crisponi syndrome; cold-induced sweating; hyperthermia; CRLF1

\section{INTRODUCTION}

Mutations in CRLF1 (cytokine receptor-like factor 1) account for both Crisponi syndrome (CS; MIM\#601378) and cold-induced sweating syndrome type 1 (CISS1; MIM\#272430).

Crisponi syndrome was initially described in 1996 in 17 patients from 12 different families in southern Sardinia. ${ }^{1}$ Further patients have been reported later. ${ }^{2-8}$ The syndrome usually manifests at birth, when patients present with hyperthermia and abnormal paroxysmal contractions of the facial and oropharyngeal muscles, as well as feeding and respiratory difficulties often requiring the use of nasogastric feeding. Physical dysmorphisms such as a large face, broad nose and camptodactyly have been described in most of the patients. ${ }^{3-5,7,8}$ Hyperthermia is frequently associated with death within the first months of life. Feeding difficulties and hyperthermia often resolve after infancy in the rare surviving patients, who then develop scoliosis and sometimes psychomotor retardation. In pre-adolescent patients, evidence of cold-induced sweating was reported. ${ }^{3}$

Cold-induced sweating syndrome type 1 (CISS1) was first reported in two Israeli sisters in $1978 .^{9}$ It involves paradoxical sweating at cold ambient temperatures on the upper part of the body, along with progressive scoliosis. In both sisters, dysmorphic features including a high arched palate, nasal voice and joint contractures have been observed. Two Norwegian brothers and a Canadian woman with a similar phenotype have been described more recently. ${ }^{10,11} \mathrm{CS}$ and CISS1 belong to a group of genetic disorders with similar phenotypes associated with mutations of genes in the ciliary neurotrophic factor receptor (CNTFR) pathway, which is known to be important for the development and maintenance of the nervous system and muscles. ${ }^{12}$ This group includes cold-induced sweating syndrome type 2 (CISS2; MIM\#610313), caused by mutations in $C L C F 1,{ }^{10,13}$ and Stüve-Wiedemann syndrome (SWS; MIM\#601559), caused by mutations in LIFR. ${ }^{14}$ Mutations in CLCF1 lead to a phenotype similar to mutations in CLRF1, with cold-induced sweating, scoliosis and cubitus valgus. ${ }^{10,13}$ Clinical features of SWS also include camptodactyly, feeding difficulties, scoliosis and temperature instability, also present in the other syndromes, but the characteristic bowing of the long bones is not present in CS, CISS1 and CISS2. ${ }^{14-19}$ Clinical similarities between Crisponi syndrome and cold-induced sweating syndrome type 1, along with the involvement of the same gene (CRLF1), led to the question whether these two syndromes represent two allelic diseases or, in fact, manifestations of the same disorder, reported at different ages of affected patients. ${ }^{1,2}$

${ }^{1}$ Department of General Pediatrics, Münster University Children’s Hospital, Münster, Germany; ${ }^{2}$ Istituto di Neurogenetica e Neurofarmacologia, Consiglio Nazionale delle Ricerche (INN-CNR), c/o Cittadella Universitaria di Monserrato, Cagliari, Italy; ${ }^{3}$ Dipartimento di Scienze Biomediche e Biotecnologie, Università degli Studi di Cagliari, Cagliari, Italy; ${ }^{4}$ Hospital Universitario La Paz Neurologia Pediatrica, Paseo de la Castellana 261, Madrid, Spain; ${ }^{5}$ stituto di Clinica Pediatrica, Università Cattolica del Sacro Cuore, Roma, Italy; ${ }^{6}$ Department of Genetic Medicine and Development, Geneva University Hospital, Geneva, Switzerland; ${ }^{7}$ Arab Medical Center, Department of Pediatrics, Amman, Jordan; ${ }^{8}$ Section for pediatrics, Department of Clinical Medicine, University of Bergen, Bergen, Norway; ${ }^{9}$ Casa di cura Sant'Anna, Cagliari, Italy

*Correspondence: Dr L Crisponi, Istituto di Neurogenetica e Neurofarmacologia, Consiglio Nazionale delle Ricerche (INN-CNR), c/o Cittadella Universitaria di Monserrato, Strada statale 554 bivio per Sestu km 4500, Monserrato (Cagliari) 09042, Italy. Tel: +39 070675 4591; Fax: +39 070 675 4652; E-mail: laura.crisponi@inn.cnr.it

${ }^{10}$ These authors contributed equally to this work.

Received 14 July 2010; revised 13 December 2010; accepted 14 December 2010; published online 16 February 2011 
In an effort to delineate the specific clinical features attributed to CS and CISS1, we investigated the clinical history, physical characteristics and experimental data of 19 patients with mutations in CRLF1, 14 of them classified as CS ( 9 reported and 5 unreported cases) and 5 as CISS1 (all reported), ${ }^{9-11}$ by means of a standardized questionnaire.

Because a genotype/phenotype correlation has not been found in previous studies, ${ }^{2,3}$ we attempted to carry out a detailed analysis of the clinical phenotype and compare it with the genetic and functional data from the investigation of the CRLF1 gene and protein for all the probands analyzed.

We found that phenotypic severity of CRLF1-associated disorders depends on altered kinetics in the secretion of the mutated CRLF1 protein, suggesting that CS and CISS1 are manifestations of the same disease.

\section{PATIENTS AND METHODS}

\section{Patients and clinical data}

Inclusion in the study was based on the diagnosis of CS or CISS1 with proven mutations on both alleles of the CRLF1 gene. Diagnosis of either syndrome was established on the basis of compilation of symptoms: $\sim 14$ patients diagnosed with CS and 5 patients diagnosed with CISS1 were included in the study. The 14 patients classified as CS (9 females, 5 males) originated from 11 families from Italy, Turkey, Spain and Libya (Table 1). The five patients (3 females, 2 males) classified as CISS1 originated from three families from Norway, Canada and Israel and showed a heterogenic phenotype (Table 1). The study protocol was approved by the Münster University Hospital Ethical Committee in Germany and all subjects involved in this study gave informed written consent.

Clinical data and patient history were gathered through a standardized questionnaire, which was sent to the responsible clinicians, and through review of the clinical data presented in the literature. Data on facial and skull height, as well as nasal and philtrum length, were obtained from a subset of probands; measurements were plotted according to the Handbook of Normal Physical Measurements, $1989 .{ }^{20}$

\section{DNA extraction and mutation analysis}

Genomic DNA was isolated from peripheral blood by a salting-out protocol. All the nine coding exons and surrounding intronic regions of CRLF1 (GenBank accession number NM_004750U) were amplified by PCR using specific primers, ${ }^{3}$ and the amplicons subsequently analyzed by direct sequencing (ABI3130XL, Applied Biosystems, Carlsbad, CA, USA).

\section{DNA cloning}

The clone pCMV6-XL5CRLF1 (Origene cat.TC126412NM_004750.2) was used to subclone the cDNA encoding the human CRLF1 in the PEF5HA vector (provided by Dr Nunzio Bottini, La Jolla Institute for Allergy and Immunology, CA, USA) using EcoRI and XbaI restriction enzymes. The clone pCMV6XL5CLCF1 (Origene cat.TC122789NM_013246.2) was used to co-transfect with PEF5HA-CRLF1, both wild type and mutated, in COS-7 cells.

\section{CRLF1 site-directed mutagenesis}

The PEF5HA-CRLF1 clone was subjected to site-directed mutagenesis using the QuickChange site-directed mutagenesis kit (Stratagene Europe, Amsterdam, The Netherlands) according to the manufacturer's instructions. Primer sequences are available on request.

\section{Cell culture}

COS-7 cells were maintained in Dulbecco's Modified Eagle's Medium (DMEM; Gibco Invitrogen, Carlsbad, CA, USA) supplemented with $10 \%$ fetal bovine serum, $100 \mathrm{U} / \mathrm{ml}$ penicillin, $100 \mu \mathrm{g} / \mathrm{ml}$ streptomycin and 2-mM glutamine (Gibco Invitrogen). IMR32 neuroblastoma cell lines (DSMZ ACC165) were grown in RPMI 1640 (Gibco Invitrogen) supplemented with 10\% fetal bovine serum, $100 \mathrm{U} / \mathrm{ml}$ penicillin, $100 \mu \mathrm{g} / \mathrm{ml}$ streptomycin, 2-mm glutamine, $1 \%$ essential amino acids and 1\% sodium pyruvate (Gibco Invitrogen).

\section{DNA transfection and cell lysis}

COS-7 cells were transfected using Lipofectamine 2000 reagent (Invitrogen) according to the manufacturer's instructions and after $24,36,48$ and $72 \mathrm{~h}$ were lysed with lysis buffer $(150 \mathrm{~mm} \mathrm{NaCl}, 50 \mathrm{~mm}$ TrisHCl pH 7.5, 1 mм EDTA, $1 \%$ Triton X-100, $0.1 \%$ sodium deoxycholate, $4 \mathrm{~mm}$ pefablock) containing Complete Protease Inhibitor Cocktail (Roche, Basel, Switzerland).

\section{Western blotting}

Protein concentrations were determined by a spectrophotometer using Bio-Rad Protein Assay (Bio-Rad Laboratories, Segrate, Italy). Thereafter, $50 \mu \mathrm{g}$ of lysates and $30 \mu \mathrm{l}$ of supernatants were subjected to SDS-PAGE with 9\% polyacrylamide gels and transferred to PVDF membrane Hybond-P (Amersham, GE Healthcare Europe GmbH, Milano, Italy) by electroblotting. The membranes were blocked in PBS with $1 \%$ Tween 20 (PBS-T) and 5\% of milk for $1 \mathrm{~h}$ at room temperature and then incubated with anti-CRLF1 (mouse monoclonal Abcam ab26125) or anti-CLCF1 (rabbit polyclonal Abcam ab56500) antibody overnight at $4^{\circ} \mathrm{C}$, at a dilution of 1:500 and 1:1000, respectively. The membranes were washed three times for $10 \mathrm{~min}$ in PBS-T and incubated in the appropriate secondary antibody for $1 \mathrm{~h}$ at room temperature. After washing, the blots were developed by chemiluminescence using ECL plus reagent (Amersham Biosciences), according to the manufacturer's instructions. To carry out densitometric analysis to evaluate CRLF1 secretion level, we used Image J software (http://rsbweb.nih.gov/ij/). We defined a rectangular area surrounding each band, and keeping this area constant we measured the corresponding mean of intensities. We then subtracted the values of background area and calculated the percentage rate related to the mean of the intensities between supernatants and lysates. The level of wild-type CRLF1 secretion in the supernatants was considered as $100 \%$. The result is representative of at least three independent experiments.

\section{Tyrosine phosphorylation analysis}

After a $24 \mathrm{~h}$ serum starvation, IMR32 cells were stimulated for $10 \mathrm{~min}$ with the medium recovered from COS-7 cells co-transfected with wild type or mutant CRLF1 and CLCF1, with serum-free medium as negative control and with $100 \mathrm{pm}$ human recombinant ciliary neurotrophic factor expressed in E. coli (C3710 no. 086k1965, Sigma-Aldrich Corp., St Louis, MO, USA) in serum-free medium as positive control. The cells were then lysed with lysis buffer $(150 \mathrm{~mm}$ $\mathrm{NaCl}, 50 \mathrm{~mm}$ TrisHCl pH 7.5, $1 \mathrm{~mm}$ EDTA, 1\% Triton X-100, 0.1\% sodium deoxycholate and $4 \mathrm{~mm}$ pefablock) containing Complete Protease Inhibitor Cocktail (Roche) and HaltPhosphataseInhibitor Cocktail (Pierce Biotechnology, Inc., Rockford, IL, USA). Thereafter, $50 \mu \mathrm{g}$ of lysates were subjected to SDS-PAGE analysis with $9 \%$ polyacrylamide gel and transferred to PVDF membrane Hybond-P (Amersham) by electroblotting. The filter was immunoblotted with an antibody specific for all the phospho-STAT3 isoforms (Cell Signaling Technology Inc., Danvers, MA, USA, no. 9131s, dilution 1:1000). The membrane was then stripped in $0.1 \mathrm{~N} \mathrm{NaOH}$ and reblotted with an antibody specific for STAT3 (Cell Signaling, no. 9132, dilution 1:1000).

\section{RESULTS}

Clinical features of patients classified with Crisponi syndrome and CISS1 syndrome

In our study, patients classified as CS presented with typical symptoms in infancy, including severe hyperthermia, contraction of facial muscles, feeding difficulties with hypersalivation, which often required nasogastric feeding, and dysmorphic features, for example, camptodactyly, foot anomalies, high arched palate and chubby cheeks (Figure 1, Table 1 and Supplementary Table 1). The severe manifestations led to death in most of the historically described cases.

However, these classical symptoms of CS could be observed in some patients with the established diagnosis of CISS1 as well (Figure 2, Table 1 and Supplementary Table 1). The Norwegian brothers described initially by Knappskog et al ${ }^{11}$ feature a phenotype that shows considerable resemblance to Crisponi patients. Feeding difficulties and the need of a nasogastric tube during infancy were 


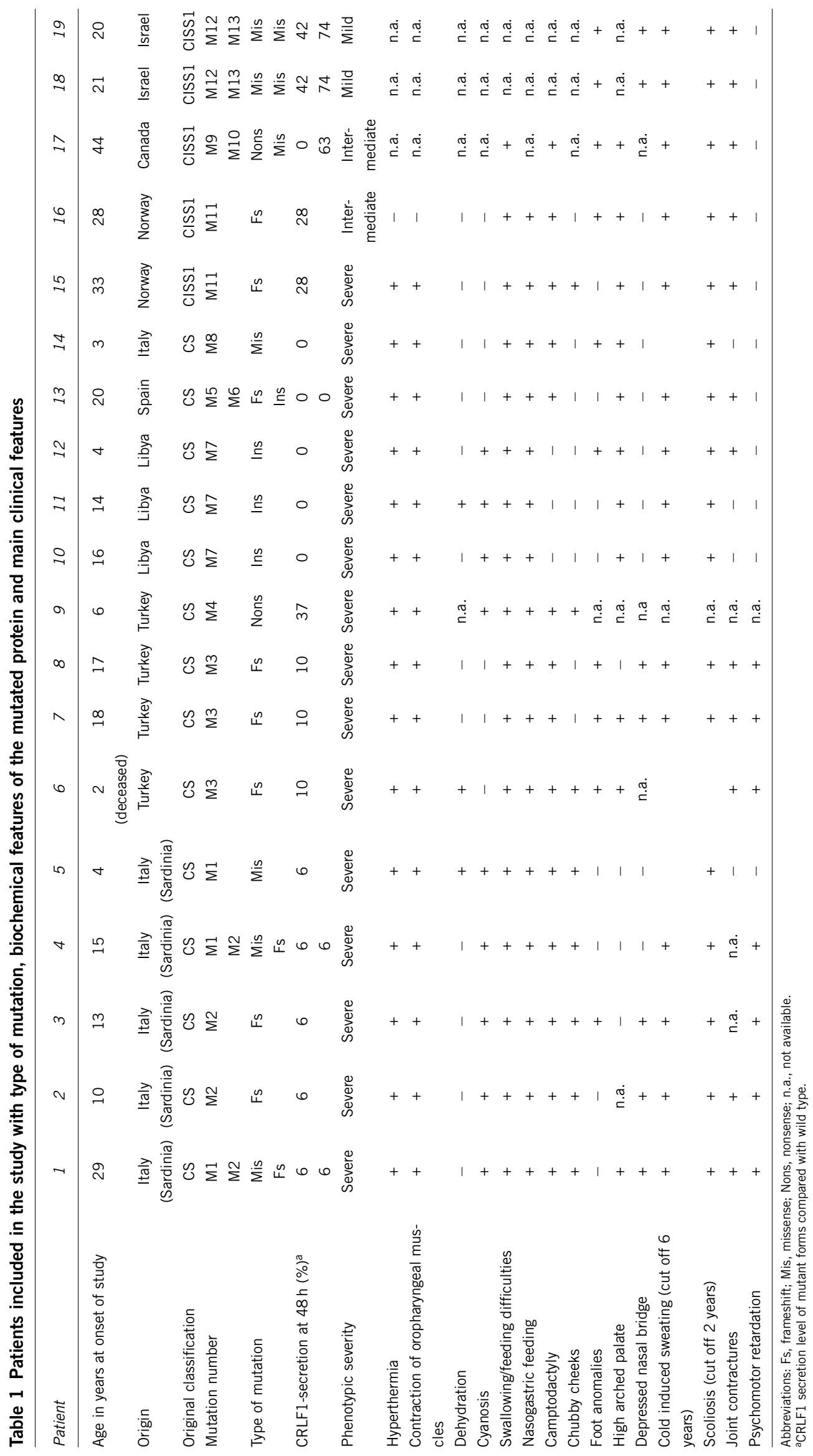




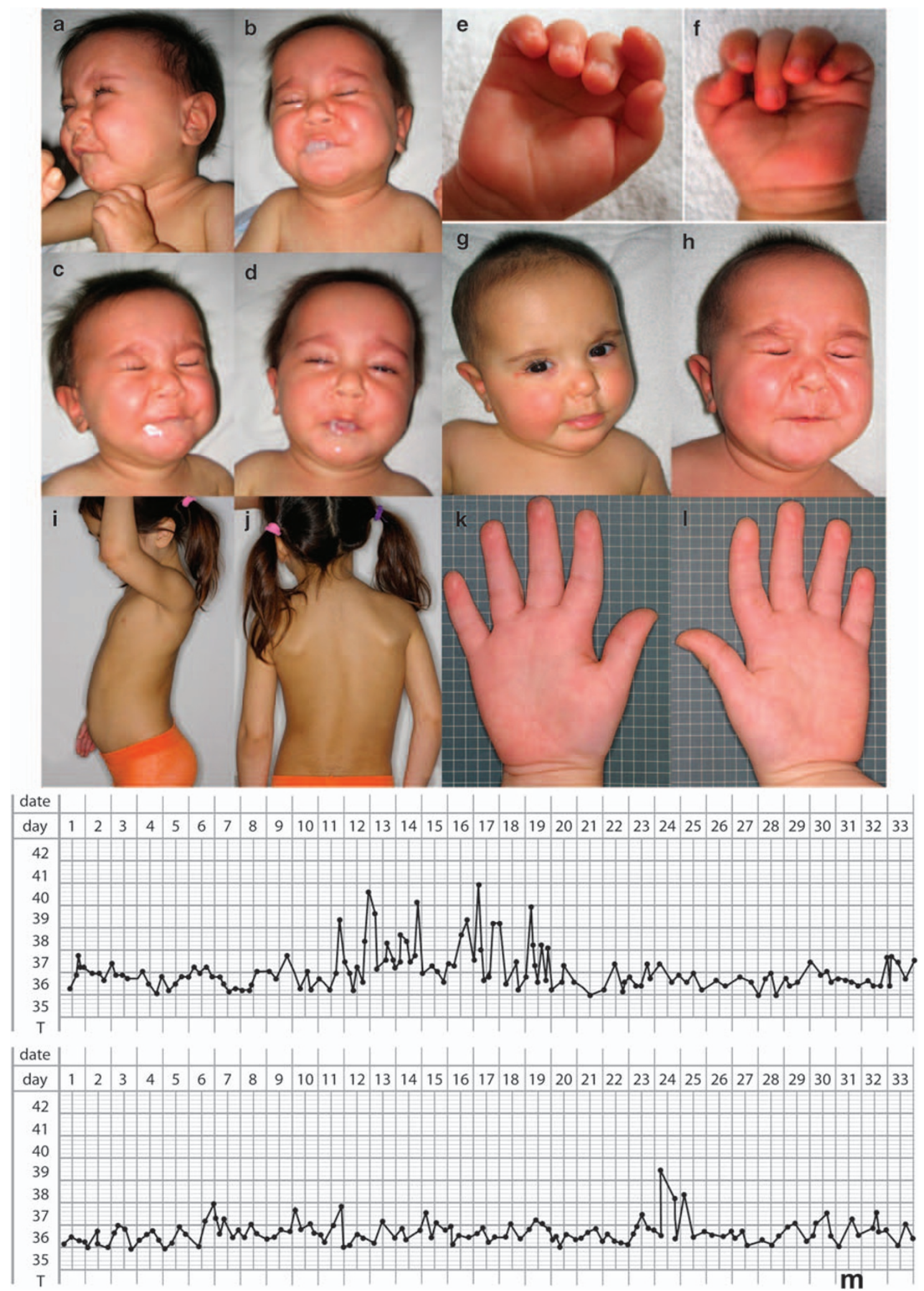

Figure 1 Clinical characteristics of patients classified as having Crisponi syndrome. (a-d) Patient 5, at 7 months of age, showing contractions of facial muscles, chubby cheeks and hypersalivation. (e and $\mathbf{f}$ ) Patient 5 at 1 year of age with camptodactyly of both hands. (g and $\mathbf{h}$ ) Patient 5 at 1 year of age with contractions of facial muscles (h). (i and j) Patient 5 at 5 years and 4 months of age, showing hyperkyphosis (i) and scoliosis (j). (k and I) Patient 5 at 5 years and 4 months of age with camptodactyly of both hands. (m) Typical fever course of a patient with Crisponi Syndrome (Patient 2) after birth. Graphs show the core temperature during 66 days of measurement with peaks up to $41^{\circ} \mathrm{C}$. 

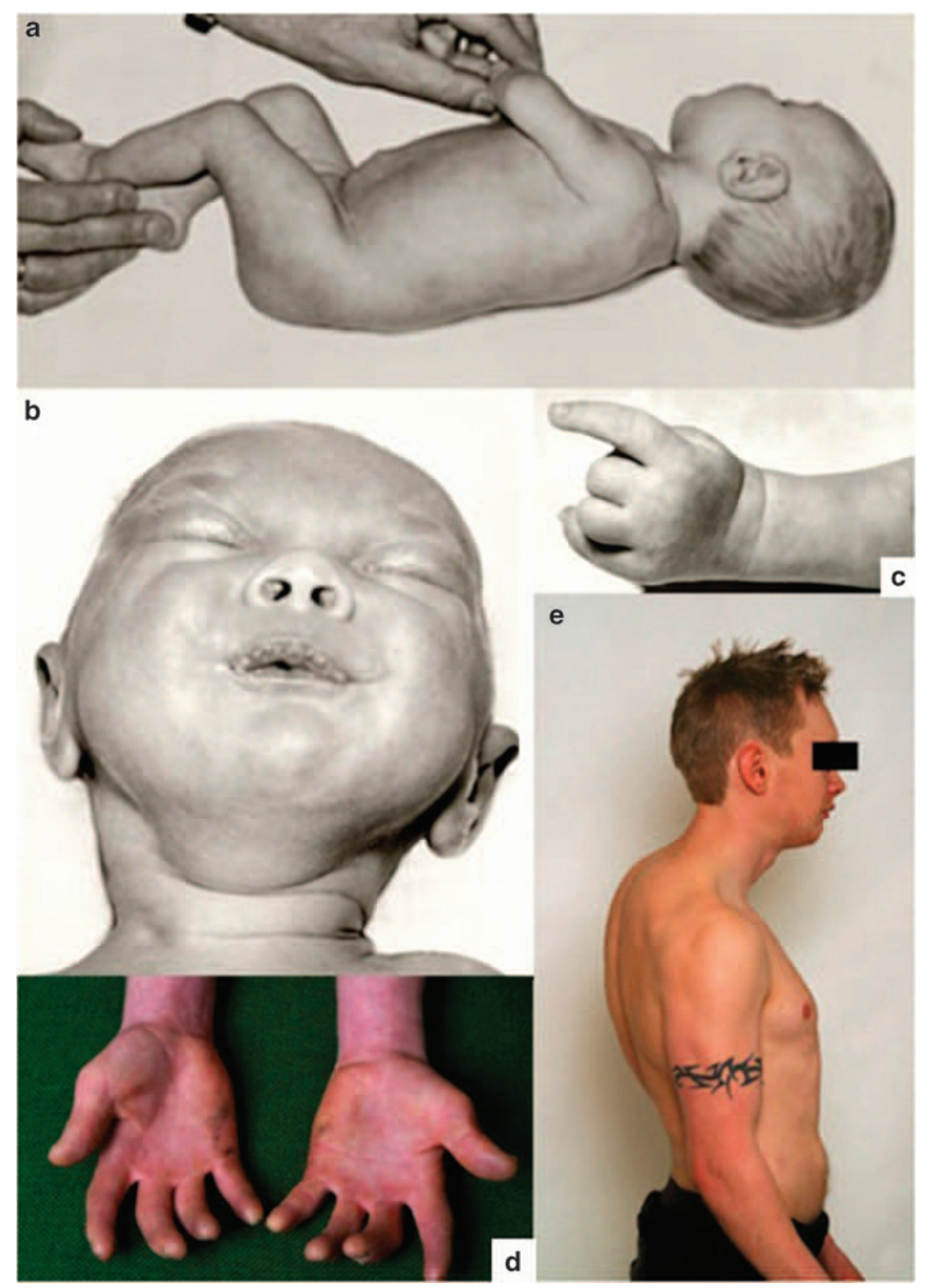

Figure 2 Clinical characteristics of two Norwegian patients classified as having CISS1. (a) Patient 15, in infancy, presenting contractures in elbows and knees. (b) Patient 15, showing ocular and oropharyngeal contractions and chubby cheeks. (c) Patient 15, with typical camptodactyly of the hand. (d) Patient 16 , at the age of 18 years, with camptodactyly of both hands. (e) Patient 16, at the age of 18 years, presenting micrognathia, kyphosis, scoliosis and flexion contractures of the elbows.

described in the paper, whereas in one of the two brothers episodes of hyperthermia in the first period of life were reported only later during a reconsideration of the cases for this study. Further, the eldest brother showed contractions of facial muscles in infancy (Figure 2b). Feeding difficulties in infancy were also described in the Canadian woman. ${ }^{10}$

Physical measurements recorded in 14 patients with CRLF1 mutations did not reveal a significant difference in facial attributes in comparison with the general population (data not shown). These observations show, in contrast to previous studies, ${ }^{1}$ that the attribute of a long and large face, long philtrum and broad nose cannot be included in the obligatory features of patients with CRLF1 mutations.

All surviving patients older than 2 years in our study cohort developed scoliosis, and those older than 6 years developed paradoxical sweating in response to low ambient temperatures. Some patients had been treated with Clonidine to reduce sweating. Patients 15 and 18 showed long lasting positive effects, whereas patient 16 showed no significant improvement and preferred physical training as a method against sweating. ${ }^{10}$ Patients 17 and 19 showed positive but decreasing effects over time. ${ }^{10}$ Further, patients 7 and 8 , originally classified as CS patients, responded to $\alpha$-adrenergic agents with regard to paradoxical sweating. ${ }^{21}$

Diagnostic studies such as electroencephalogram, cranial computed tomography, muscle biopsy and nerve conduction velocity were carried out in several cases mostly showing normal results (Supplementary Table 2). Most of the initial symptoms resolved after the first 1 or 2 years of life. Some patients still showed hypersalivation or hyperthermia in adulthood (Table 1 and Supplementary Table 1). 
Our cohort contains a subgroup of patients with a mild phenotype in early childhood presenting no hyperthermia or feeding difficulties after birth (patients 17-19, Table 1). In pre-adolescence, these patients developed scoliosis and cold-induced sweating (Table 1).

\section{CRLF1 mutational analysis}

The CRLF1 gene (GenBank accession number NM_004750) is localized on human chromosome $19 \mathrm{p} 13.11$ and is composed of nine coding exons (Figure 3a). The encoded protein (GenBank accession number NP_004741) comprises 422 amino acids. By direct sequencing of all the nine coding exons, as well as exonic/intronic boundaries of the CRLF1 gene, different types of mutations have been identified in all the patients included in this study (Figure 3, Table 1, Table 2). Six patients (Table 1) were compound heterozygotes for different mutations, and the remaining were homozygotes for one type of mutation. No obvious correlation between the type/localization of mutation and the severity of the clinical course was detected (Table 1).

\section{CRLF1 functional analysis}

CRLF1 protein is known to be a member of the ciliary neurotrophic factor receptor (CNTFR) pathway by forming a complex with CLCF1. The stable secreted complex of CRLF1 and CLCF1 forms a ligand for CNTFR $\alpha$, which, along with gp130 and LIFR, compose the
CNTF-receptor complex. ${ }^{22}$ CRLF1/CLCF1 binding to CNTFR $\alpha$ leads to dimerization of gp130/LIFR, which then induces downstream signaling events, including activation of the JAK1/STAT3 pathway. ${ }^{22}$ To investigate the biological significance and to assess the secretion capacities of mutated CRLF1, mutations found in the 19 patients (Table 1) were introduced into wild-type CRLF1 cDNA and co-transfected with wild-type CLCF1 cDNA in COS-7 cells. Non-transfected COS-7 cells were used as controls.

After a $48 \mathrm{~h}$ culture period, strong expression of wild-type CRLF1 and mutants was detected in cell lysates, whereas only wild-type CRLF1, mutants K368X (M4), W284C (M10), R81H (M12) and L374R (M13) were strongly detected in the supernatant of transfected cells. CRLF1 mutants W76G (M1), T226NfsX104 (M2), P238RfsX6 (M3) and V282GfsX47 (M11) were only partially secreted into the medium, whereas Y75D (M5), P239AfsX92 (M6), D181GfsX5 (M7), [N113I;L114P] (M8) and Q180X (M9) were not secreted or detected at all (Supplementary Figure 1a, Table 2).

Previous studies reported that CLCF1 must interact with CRLF1 in the 'producer' cell to be released. ${ }^{23}$ To evaluate the ability of mutated CRLF1 to stimulate CLCF1 secretion, we co-transfected wild type and mutant forms of CRLF1 together with wild-type CLCF1. After a $48 \mathrm{~h}$ culture period, we found CLCF1 in culture supernatants when transfected alone, as well as when co-transfected with the wild type

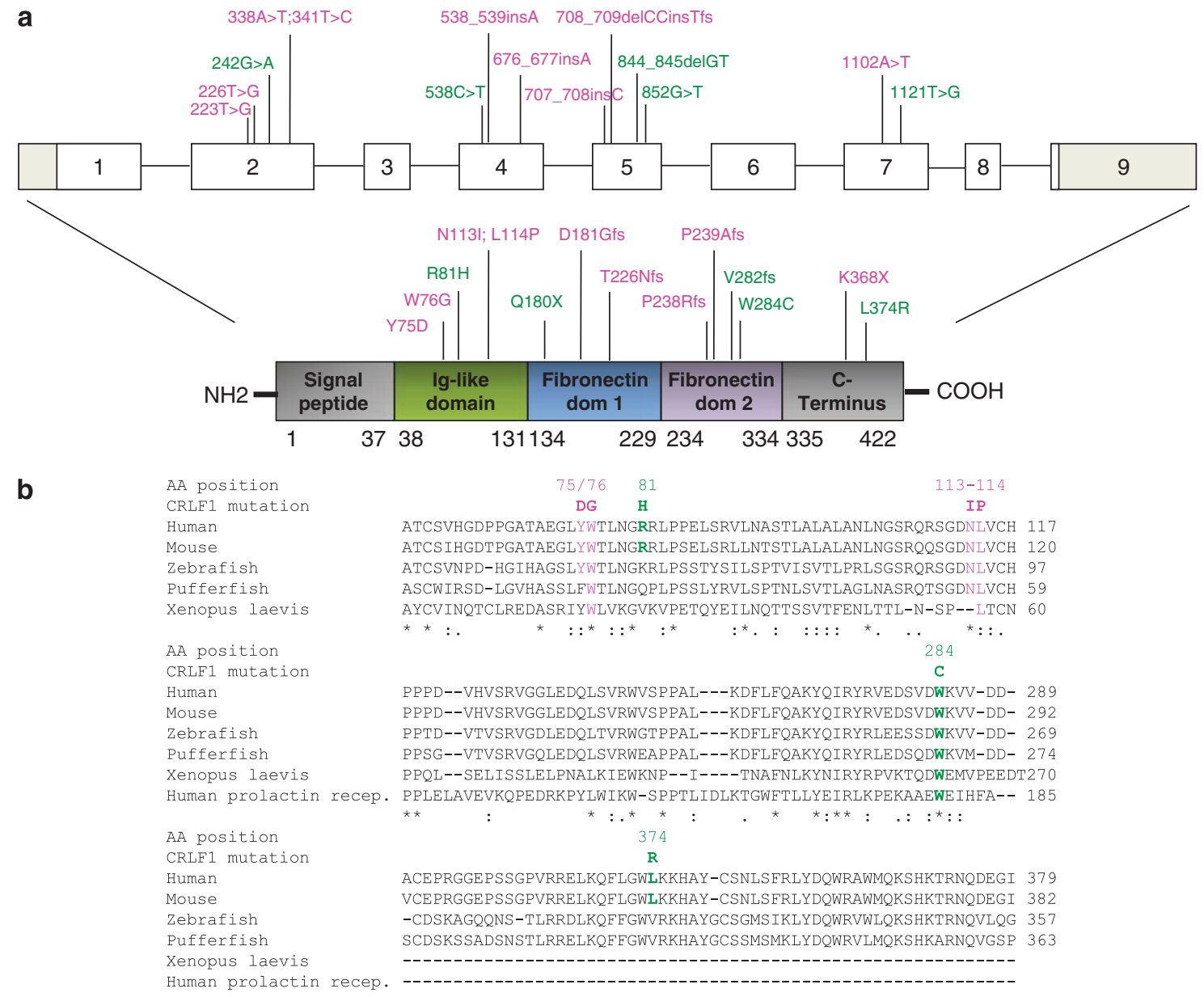

Figure 3 Structure of the human CRLF1 gene and protein. (a) Relative positions of the mutations found in both CS and CISS1. (b) Alignment and aminoacid conservation for both Crisponi and CISS syndrome missense mutations analyzed. SIFT (Sorting Intolerant From Tolerant; http://sift.jcvi.org/) predicted that mutations Y75D, W76G, [N113I;L114P], W284C and L374R are not tolerated, whereas R81H is tolerated. Pink: CS mutations, green: CISS1 mutations. 
Table 2 CRLF1 mutations found in CS and in CISS1 patients and functionally analyzed

\begin{tabular}{|c|c|c|c|c|c|c|c|}
\hline Mutation number & Origin & CRLF1 exon & DNA sequence variant & Amino acid variant & Mutation type & Syndrome & CRLF1 secretion (\%) \\
\hline M1 & Sardinia (Italy) & 2 & c. $226 \mathrm{~T}>\mathrm{G}$ & p.W76G & Missense & $\operatorname{cS}^{3}$ & 6 \\
\hline M2 & Sardinia (Italy) & 4 & c.676-677insA & p.T226NfsX104 & Frameshift & $\mathrm{CS}^{3}$ & 6 \\
\hline M3 & Turkey & 5 & c.708-709delCCinsT & p.P238RfsX6 & Frameshift & $\mathrm{CS}^{3}$ & 10 \\
\hline M4 & Turkey & 7 & c. $1102 \mathrm{~A}>\mathrm{T}$ & p.K368X & Nonsense & $\operatorname{cs}^{3}$ & 37 \\
\hline M5 & Spain & 2 & c. $223 \mathrm{~T}>\mathrm{G}$ & p.Y75D & Missense & CS & 0 \\
\hline M6 & Spain & 5 & c.713dupC & p.P239AfsX92 & Insertion & CS & 0 \\
\hline M7 & Libya & 4 & c.538_539insA & p.D181GfsX5 & Insertion & CS & 0 \\
\hline M8 & Italy & 2 & c. $[338 \mathrm{~A}>\mathrm{T} ; 341 \mathrm{~T}>\mathrm{C}]$ & p.[N113I;L114P] & Missense & CS & 0 \\
\hline M9 & Canada & 4 & c. $538 \mathrm{C}>\mathrm{T}$ & p.Q180X & Nonsense & CISS $1^{10}$ & 0 \\
\hline M10 & Canada & 5 & c. $852 \mathrm{G}>\mathrm{T}$ & p.W284C & Missense & CISS $1^{10}$ & 63 \\
\hline M11 & Norway & 5 & c.844-845delGT & p.V282GfsX47 & Frameshift & CISS $1^{11}$ & 28 \\
\hline M12 & Israel & 2 & c. $242 \mathrm{G}>\mathrm{A}$ & p.R81H & Missense & CISS $1^{11}$ & 42 \\
\hline M13 & Israel & 7 & c. $1121 \mathrm{~T}>\mathrm{G}$ & p.L374R & Missense & CISS $1^{11}$ & 74 \\
\hline
\end{tabular}

and mutant CRLF1, although with different signal intensities (Supplementary Figure 1b). To better understand these findings, we carried out a time-course experiment across different culture periods (24, 36, 48 and $72 \mathrm{~h})$ for both wild-type CRLF1 and CLCF1 transfected alone or together in COS-7 cells. Non-transfected COS-7 cells were used as control (Supplementary Figure 2a, b). The results indicate that, in the cell lysate, CLCF1 alone is always expressed from 24 to $72 \mathrm{~h}$, whereas in supernatants it is detected at 48 and $72 \mathrm{~h}$. When co-transfected with CRLF1, CLCF1 was only faintly detected in supernatants at $24 \mathrm{~h}$, whereas it was strongly detected at 36,48 and $72 \mathrm{~h}$. Thus, it seems that coexpression with CRLF1 is not necessary for CLCF1 secretion, but is required to accelerate the kinetics of secretion into the extracellular medium.

\section{Analysis of induction of STAT3 tyrosine phosphorylation by the CRLF1/CLCF1 complex}

It has been previously reported that the secreted heterodimer CRLF1/ CLCF1 activates the CNTF receptor with the induction of downstream signaling events, including activation of the JAK1/STAT3 pathway. ${ }^{22,24}$ Therefore, we assessed the ability of CRLF1 mutants to induce STAT3 signal transduction in the IMR32 neuroblastoma cell line, expressing the CNTF receptor complex (gp130, LIFR $\beta$ and CNTFR $\alpha) .{ }^{22}$ The supernatants of COS-7 cells transfected with wild-type CLCF1 or wild-type CRLF1 alone, and with wild-type CLCF1 and wild type or mutant forms of CRLF1, were added to an IMR32 cell line after $24 \mathrm{~h}$ serum starvation. CNTF was used as a control at a concentration of 100 pM. After a $10 \mathrm{~min}$ incubation, cells were lysed and analyzed by western blotting with anti-STAT3 and anti-phosphoSTAT3 antibodies. We found that CLCF1 alone can induce STAT3 phosphorylation in an IMR32 cell line, but CRLF1 alone cannot (data not shown). However, the complexes of wild-type CLCF1 and wild-type or mutated forms of CRLF1 were able to elicit STAT3 signaling.

\section{DISCUSSION}

\section{Clinical and diagnostic considerations}

In this study, we collected and analyzed in detail clinical data from 19 patients diagnosed with CS or CISS1. Patients with mutations in the CRLF1 gene until now have been classified as CS or CISS1 on the basis of the severity of their clinical symptoms and the age at diagnosis. However, a detailed analysis of the clinical phenotypes in this study showed that there is a clinical course of varying severity in both groups of patients. The Israeli sisters described in 1978 by Sohar et al ${ }^{9}$ exhibited a less-severe phenotype and have formed the entity of CISS1. Further patients such as the Norwegian brothers have been included in this group. However, our retrospective study showed that they presented symptoms in early childhood including feeding difficulties and hyperthermia, which have been reported only for CS. On the other hand, CS patients who have survived the critical first months of life develop typical symptoms of CISS1 as they grow older. This makes it very likely that the initial CS and CISS1 patients reported in the paper by Crisponi ${ }^{1}$ and by Sohar et al ${ }^{9}$ have been considered as different entities only because the patients were reported at different ages. This is in line with a most recent study reporting a CISS1 patient with neonatal features of Crisponi syndrome, supporting the notion that CISS1 and CS may be a single clinical entity. ${ }^{25}$

The overlapping phenotypes (Table 1 and Supplementary Table 1) make it difficult at present to classify new patients. To elucidate a potential genotype/phenotype correlation, we carried out a detailed analysis in relation to the mutations found in the CRLF1 gene.

\section{Classification according to the type of mutation}

The different types of mutations were analyzed for all patients included in this study. Missense, nonsense, insertions and frameshift mutations were found. Strikingly, patients who have suffered a severe clinical course with hyperthermia and feeding difficulties during childhood display different kinds of mutations. The deceased patients do not differ from other patients with regard to the type of mutation. Further, the same class of mutation is responsible for different clinical courses, as exemplified by patient 5 , who displays an extremely severe phenotype until now, and patients 18 and 19, who are known to have shown a very mild clinical course. As missense mutations were detected in all three of them, a strict correlation between the type of mutation and the phenotype seems very unlikely. The variety of mutations leading to the same phenotype and the variety of phenotypes caused by the same type of mutation lead to the conclusion that there is no strict correlation between phenotype and genotype based on the type/localization of CRLF1 mutation.

\section{Classification according to the biochemical characteristics of CRLF1}

We also carried out a functional analysis of the mutated forms showing that different mutations can be grouped according to their biochemical quality. In the first group, absent or weak secretion 
(less than $40 \%$ compared with wild type) of mutant CRLF1 occurred; in the second group, the mutated protein was strongly secreted (more than $40 \%$ compared with wild type, Table 1, Table 2).

Patients 1-15 are carriers of mutations belonging to the first group. The clinical phenotype was severe for all patients in this group (Table 1). All these patients showed hyperthermia, contraction of facial muscles, hypersalivation and feeding difficulties in early childhood and required nasogastric feeding. All patients older than 2 years developed scoliosis; those older than 6 years also developed cold-induced sweating.

Patients 18 and 19 carry mutations associated with a strong CRLF1 secretion. These patients described by Sohar et al ${ }^{9}$ exhibit a mild phenotype without severe manifestations in infancy, such as hyperthermia, but with cold-induced sweating, scoliosis and some dysmorphic features. On the basis of these observations, we conclude that phenotypic severity of CRLF1-associated disorders mainly depends on altered secretion of the CRLF1 protein. Accordingly, patient 17, who is a compound heterozygous carrier of two mutations, associated with absent and strong CRLF1 secretion, respectively, presents an 'intermediate' phenotype with feeding difficulties in infancy, and scoliosis and cold-induced sweating in adolescence.

However, it is likely that in some cases the severity of the phenotype could also be affected by other factors. For example, in patient 16, genetic factors may account for the milder phenotype, compared with his brother (patient 15) who carries the same mutation.

Our analysis supports the conclusion that absent or weak CRLF1 secretion is associated with a severe clinical phenotype, whereas strong secretion is associated with a milder clinical phenotype. On the basis of the fact that our retrospective clinical study was limited either by the small number of patients in each CRLF1-secretion-based group or by clinical information that is not exhaustive, we suggest that defective secretion is a major component affecting phenotypic severity of CRLF1-associated disorders.

\section{Analysis of the CRLF1/CLCF1 pathway}

CRLF1 protein is a member of the ciliary neurotrophic factor receptor (CNTFR) pathway by forming a complex with CLCF1, which binds to the CNTF receptor. In contrast to the observations previously reported, in which CLCF1 can be actively secreted from cells by forming a complex with CRLF1, ${ }^{22,24}$ we found that CLCF1 was detected in culture supernatants when transfected alone, as well as when co-transfected with wild type and mutant CRLF1, although with different signal intensities (Supplementary Figure 1b).

It is noteworthy that an alteration in the kinetics of CLCF1 secretion is associated with coexpression with some mutant forms of CRLF1, which may inhibit its secretion into the medium. Further studies aiming to identify the CRLF1 interaction sites with CLCF1 will help to better understand the heterodimer complex formation and the role of the CRLF1 mutations analyzed.

It has been previously reported that the secreted heterodimer CRLF1/CLCF1 activates the CNTF receptor with the induction of downstream signaling events, including activation of the JAK1/STAT3 pathway. Dagoneau et al ${ }^{2}$ already reported that STAT3 phosphorylation was normally triggered in fibroblasts of patients with Crisponi syndrome by LIF. We found the same result in our experiments (data not shown). Although CRLF1 is not needed to activate the CNTF receptor, early secretion of the full CRLF1 protein seems to be necessary for adequate CLCF1 secretion and proper activation of the CNTF-receptor pathway.

However, the results from the cell culture experiments cannot be directly transferred to the mechanisms in humans; hence, it cannot be fully estimated how a delay of $48 \mathrm{~h}$ in a cell culture would affect function in organ systems. Alternatively, another receptor may be involved, and the alteration of its pathway may cause the phenotype. This hypothesis gains some evidence from the recent detection of a previously unknown receptor for CRLF $1 .^{26}$

Our findings lead to the old matter about 'lumpers' and 'splitters' ${ }^{27}$ and thus to the critical question of how to classify and name these genetic entities. In the presence of bowing of the long bones, the pediatric manifestation would lead to Stüve-Wiedemann syndrome. ${ }^{28}$ In the absence of bowing of the long bones, on the basis of our findings, we believe that the two syndromes, CS and CISS1, represent manifestations of one disorder, with different degrees of severity. The rare cases of CISS1 most likely correspond to CS survivors. Genetic heterogeneity could be assumed with CISS2, which shows the same phenotype as CISS1, but is due to mutations in CLCF1. However, as there has been only one case described in literature, this assumption might be too early at the moment.

Use of one facet of the syndrome, such as cold-induced sweating as a name for the whole, has some limitations, as this feature is not the only one, may occur as an isolated anomaly or may be a feature of other syndromes. Therefore, we suggest renaming the two genetic entities CS and CISS1 with the broader term of Sohar-Crisponi syndrome. Furthermore, we conclude that defective secretion of the CRLF1 protein is a major factor contributing to the phenotypic severity of this syndrome.

\section{CONFLICT OF INTEREST}

The authors declare no conflict of interest.

\section{ACKNOWLEDGEMENTS}

We are grateful to all the patients and their family members who with their enthusiastic, continuous and generous participation made this study possible. This work was supported by the Associazione Sindrome di Crisponi e Malattie Rare ONLUS (grant to LC) by the Regione Autonoma della Sardegna (grant to LC) and by the Interdisciplinary Center for Clinical Research, Münster, Germany (grant to FR). We thank Professor Antonio Cao, Professor Francesco Cucca and Dr David Schlessinger for the critical reading of the manuscript.

1 Crisponi G: Autosomal recessive disorder with muscle contractions resembling neonatal tetanus, characteristic face, camptodactyly, hyperthermia, and sudden death: a new syndrome? Am J Med Genet 1996; 62: 365-371.

2 Dagoneau N, Bellais S, Blanchet P et al: Mutations in cytokine receptor-like factor 1 (CRLF1) account for both Crisponi and cold-induced sweating syndromes. Am J Hum Genet 2007; 80: 966-970.

3 Crisponi L, Crisponi G, Meloni A et al: Crisponi syndrome is caused by mutations in the CRLF1 gene and is allelic to cold-induced sweating syndrome type 1. Am J Hum Genet 2007; 80: 971-981.

4 Accorsi P, Giordano L, Faravelli F: Crisponi syndrome: report of a further patient. Am J Med Genet 2003; 123A: 183-185.

5 Nannenberg EA, Bijlmer R, Van Geel BM, Hennekam RC: Neonatal paroxysmal trismus and camptodactyly: the Crisponi syndrome. Am J Med Genet A 2005; 133: 90-92.

6 Della Marca G, Barone G, Vollono C et al: Central apneas in a case of Crisponi syndrome. Sleep Med 2008; 9: 703-704.

7 Okur I, Tumer L, Crisponi L et al: Crisponi syndrome: a new case with additional features and new mutation in CRLF1. Am J Med Genet A 2008; 146A: 3237-3239.

8 Thomas N, Danda S, Kumar M et al: Crisponi syndrome in an Indian patient: a rare differential diagnosis for neonatal tetanus. Am J Med Genet A 2008; 146A: 2831-2834.

9 Sohar E, Shoenfeld Y, Udassin R, Magazanik A, Revach M: Cold-induced profuse sweating on back and chest. A new genetic entity? Lancet 1978; 2: 1073-1074.

10 Hahn AF, Jones DL, Knappskog PM, Boman H, McLeod JG: Cold-induced sweating syndrome: a report of two cases and demonstration of genetic heterogeneity. J Neurol Sci 2006; 250: 62-70.

11 Knappskog PM, Majewski J, Livneh A et al: Cold-induced sweating syndrome is caused by mutations in the CRLF1 gene. Am J Hum Genet 2003; 72: 375-383. 
12 DeChiara TM, Vejsada R, Poueymirou WT et al: Mice lacking the CNTF receptor, unlike mice lacking CNTF, exhibit profound motor neuron deficits at birth. Cell 1995; 83: 313-322.

13 Rousseau F, Gauchat JF, McLeod JG et al: Inactivation of cardiotrophin-like cytokine, a second ligand for ciliary neurotrophic factor receptor, leads to cold-induced sweating syndrome in a patient. Proc Natl Acad Sci USA 2006; 103: 10068-10073.

14 Dagoneau N, Scheffer D, Huber C et al: Null leukemia inhibitory factor receptor (LIFR) mutations in Stuve-Wiedemann/Schwartz-Jampel type 2 syndrome. Am J Hum Genet 2004; 74: 298-305.

15 Al-Gazali LI, Ravenscroft A, Feng A, Shubbar A, Al-Saggaf A, Haas D: StuveWiedemann syndrome in children surviving infancy: clinical and radiological features. Clin Dysmorphol 2003; 12: 1-8.

16 Chabrol B, Sigaudy S, Paquis V et al: Stuve-Wiedemann syndrome and defects of the mitochondrial respiratory chain. Am J Med Genet 1997; 72: 222-226.

17 Cormier-Daire V, Superti-Furga A, Munnich A et al: Clinical homogeneity of the Stuve-Wiedemann syndrome and overlap with the Schwartz-Jampel syndrome type 2. Am J Med Genet 1998; 78: 146-149.

18 Kozlowski K, Tenconi R: Stuve-Wiedemann dysplasia in a 3 1/2-year-old boy. Am J Med Genet 1996; 63: 17-19.

19 Wiedemann HR, Stuve A: Stuve-Wiedemann syndrome: update and historical footnote. Am J MedGenet 1996; 63: 12-16.

20 Hall JG, Froster-Iskenius UG, Allanson JE: Handbook of Normal Physical Measurements. Oxford: Oxford University Press, 1989.
21 Herholz J, Crisponi L, Mallick BN, Rutsch F: Successful treatment of cold-induced sweating in Crisponi syndrome and its possible mechanism of action. Dev Med Child Neurol 2010; 52: 494-497.

22 Elson GC, Lelièvre E, Guillet $C$ et al: CLF associates with CLC to form a functional heteromeric ligand for the CNTF receptor complex. Nat Neurosci 2000; 3: 867-872.

23 Plun-Favreau H, Elson G, Chabbert M et al: The ciliary neurotrophic factor receptor alpha component induces the secretion of and is required for functional responses to cardiotrophin-like cytokine. EMBO J 2001; 20: 1692-1703.

24 Perret D, Guillet $C$, Elson G et al: Two different contact sites are recruited by cardiotrophin-like cytokine $(C L C)$ to generate the CLC/CLF and CLC/sCNTFRalpha composite cytokines. J Biol Chem 2004; 279: 43961-43970.

25 Yamazaki M, Kosho T, Kawachi S et al: Cold-induced sweating syndrome with neonatal features of Crisponi syndrome: Iongitudinal observation of a patient homozygous for a CRLF1 mutation. Am J Med Genet A 2010; 152A: 764-769.

26 Crabé S, Guay-Giroux A, Tormo AJ et al: The IL-27 p28 subunit binds cytokine-like factor 1 to form a cytokine regulating NK and T cell activities requiring IL-6R for signaling. J Immunol 2009; 183: 7692-7702.

27 McKusick VA: On lumpers and splitters, or the nosology of genetic disease. Perspect Biol Med 1969; 12: 298-312.

28 Jung C, Dagoneau N, Baujat G et al: Stüve-Wiedemann syndrome: long-term follow-up and genetic heterogeneity. Clinical Genetics 2010; 77: 266-272.

Supplementary Information accompanies the paper on European Journal of Human Genetics website (http://www.nature.com/ejhg) 